\title{
Altered Circular RNA Expression in Patients with Repeated Implantation Failure
}

\author{
Lin Liu Lifei Lia,b Xiaoling Ma ${ }^{a, b} \quad$ Feng Yue ${ }^{a, b} \quad$ Yiqing Wanga,b Liyan Wang ${ }^{a, b}$ \\ Panpan Jin ${ }^{a, b} \quad$ Xuehong Zhang ${ }^{a, b}$ \\ The Reproductive Medicine Special Hospital of the 1st hospital of Lanzhou University, Lanzhou, \\ ${ }^{b}$ Key Laboratory for Reproductive Medicine and Embryo of Gansu, Lanzhou, China
}

\section{Key Words}

Endometrial receptivity • Repeated implantation failure • Circular RNAs microarray

\begin{abstract}
Background/Aims: CircRNAs play an important role in regulating gene expression and the specific role of circRNAs in the pathogenesis of repeated implantation failure remains unclear. The aim of this study is to assess the differentially expressed circRNAs in patients with repeated implantation failure. Methods: We screened circRNA expression profiles in endometrial biopsies taken from six women with repeated implantation failure and control group using circRNA microarray. Bioinformatic analyses were applied to study these differentially expressed circRNAs. Furthermore, quantitative reverse transcription polymerase chain reaction (qRT-PCR) was performed to confirm these results. Results: The data from circRNA microarrays clearly revealed that 856 unique circRNAs were significantly altered $(p<0.05)$. The up-regulated expression of hsa_circRNA_070616, hsa_circRNA_103716, hsa_circRNA_104001, hsa_circRNA_104854 and the down-regulated expression of hsa_circRNA_004183, hsa circRNA_044353, hsa_circRNA_404686 were further validated by qRT-PCR. Conclusion: this study demonstrates that a number of circRNAs were differentially expressed in patients with repeated implantation failure compared with normal controls and may offer novel molecular candidates for diagnosis and clinical treatment of embryo implantation failures.
\end{abstract}

(C) 2017 The Author(s)

Published by S. Karger AG, Basel

\section{Introduction}

Infertility affects approximately $10-15 \%$ of couples in their reproductive age worldwide [1]. In vitro fertilization (IVF) is a widely used treatment for infertile patients and the success rates of assisted reproductive technologies have improved markedly over the last decades. Nevertheless, there are still a lot of infertile women who has good-quality embryos experiences repeated implantation failure, which may cause by factors related

L. Liu and L. Li contributed equally to this work. 
to the endometrium [2,3]. Although endometrial receptivity is assessed by morphologic features currently, exploiting noninvasive diagnostic biomarkers to accompany or replace existing assessment techniques is essential to improve the early diagnostic of implantation failures. Some studies have reported the role of LincRNA and miRNA in the development and progression of endometrial cancer and endometriosis [4-7], however, only a few studies focus on the relationship between non-coding RNA and endometrial receptivity of patients with repeated implantation failure.

CircRNAs, as a special new class of endogenous non-coding RNAs (ncRNAs), are covalently closed continuous loop with higher stability and tissue specific expression, making it more suitable biomarker than other RNA types [8-10]. It has been shown that circRNA regulate gene expression in mammals by sequestering miRNAs as miRNA sponge with miRNA response elements (MREs), and can regulate gene expression at the transcriptional or post-transcriptional level [11]. Growing evidence demonstrated that circRNAs involved in the development of several types of diseases [12-15]. Especially, Qiao and her colleagues [16] have first reported the novel profile of circRNAs in individual human oocyte and preimplantation embryos, indicating the important role of circRNA during this process. Thus, circRNAs could be considered as potential therapeutic agents or biomarkers with diagnostic abilities in the future. However, little is known about the underlying regulatory mechanisms of circRNAs with repeated implantation failure.

In the present study, the differential expression of circRNAs in endometrial biopsies taken from women with repeated implantation failure and control women with proven fertility was detected using circRNA microarray. Specifically, we identified 856 circRNAs that are up- or down-regulated in repeated implantation failure patients versus normal controls. Among them, 7 circRNAs (hsa_circRNA_070616, hsa_circRNA_103716, hsa_circRNA_104854, hsa_circRNA_104001, hsa_circRNA_004183, hsa_circRNA_044353, hsa_circRNA_404686) were confirmed by realtime qRT-PCR. These circRNAs may exhibit important function in the development and progression of repeated implantation failure. More importantly, the hsa circRNA_103716 and hsa_circRNA_070616 could be used as potential therapeutic agents and may serve as novel biomarkers with diagnostic abilities in the future.

\section{Materials and Methods}

\section{Ethics statement}

The project was approved and supervised by the Ethics Committee of the First Hospital of Lanzhou University. All sample were collected from the patient receiving operation in The First Affiliated Hospital of Lanzhou University. All patients included in this study gave their written informed consents prior to surgery.

\section{Subjects and samples}

For the control group, endometrial biopsies were obtained from fertile women who had given birth at least once. For the group to investigate, endometrial biopsies were obtained from women who had experienced repeated implantation failure after ovarian stimulation and IVF-ET. Repeated implantation failure was defined as women who had undergone $\geq 3$ failed cycles with high-grade embryos were transferred. Three pairs of snap-frozen endometrial tissue with repeated implantation failure patients and control group ( 3 normal tissues, 3 patient tissues) were collected for circRNA microarray analysis and another 6 samples (3 normal tissues, 3 patient tissues) were collected for qRT-PCR validation.

\section{RNA isolation}

Total RNA was isolated from snap-frozen endometrial biopsies of the different groups using the Trizol reagent (Invitrogen, Carlsbad, USA) following the manufacturer's instructions. The quantity and quality of the RNA samples were determined using NanoDrop ND-1000 instrument (Thermo, Wilmington, DE).

\section{CircRNA microarray hybridization}

The sample preparation and microarray hybridization were performed according to the Arraystar's standard instructions. Briefly, total RNAs were treated with Rnase R (Epicentre, Inc.), which removed linear 
RNAs to enrich circRNAs. The circRNAs were amplified and transcribed into fluorescent cRNA using random primer based on manufacturer's protocol (Arraystar Super RNA Labeling Kit; Arraystar). Next, the labeled cRNAs were hybridized onto the Arraystar Human circRNA Array V2 (8x15K, Arraystar). The hybridized arrays were then washed, fixed and scanned by the Agilent Scanner G2505C (KangChen Bio-tech, Shanghai, China).

\section{CircRNA microarray data analysis}

Agilent Feature Extraction software (version 11.0.1.1) was used to analyze acquired array images. Quantile normalization and data processing were executed using R software package. The false discovery rate (FDR) is applied to determine the threshold of p-value. An FDR $<0.05$ was recommended. CircRNAs (fold changes $\geq 2.0$ and p-values $<0.05$ ) were differentially expressed with statistical significance.

\section{Quantitative real-time polymerase chain reaction ( $q R T-P C R$ )}

Total RNA was extracted and reversely transcribed into cDNA using SuperScriptTM III Reverse Transcriptase (Invitrogen). The relative expression of circRNA related with repeated implantation failure that differentially expressed was determined by ViiA 7 Real-time PCR System (Applied Biosystems). Triplicates were performed for each sample in three independent experiments.

Bioinformatics analysis of miRNA response elements

The interactions between circRNA and miRNA were predicted with Arraystar's home-made miRNA target prediction software based on TargetScan and Miranda. The top 5 putative target miRNAs of differential expression circRNAs were identified according to seed match sequences.

\section{Statistical analysis}

Data are presented as the mean \pm standard error (SE) for triplicate measurements. The significance of the data was estimated by Student's t test. A value of $\mathrm{p}<0.05$ was considered to be statistically significant.

\section{Results}

Identification of differentially expressed circRNAs in patients with repeated implantation failure

The concentration and purity of total RNA from different samples were determined using NanoDrop ND-1000 (NanoDrop, Wilmington, USA). All RNA samples showed O.D A260/280 ratio between 1.8 and 2.1 and an 0.D A260/230 ratio >1.8.

We identified circRNAs that were differentially expressed with statistical significance between the two groups using Fold Change filtering and Volcano Plot filtering (Fig. 1A and B). Box plots showed the distribution of expression values of circRNAs, which were nearly the same after normalization (Fig. 1C).

Furthermore, we analyzed the distribution of the differential expression of circRNAs on all the human chromosomes (Fig.1D). Also, we analyzed the category of differentially expressed circRNAs. Most of them are transcribed from the protein coding exons, some are from introns and a few other sources of circRNAs (Fig. 1E).

Identification of differentially expressed circRNAs in patients with repeated implantation failure

The hierarchical clustering analysis was performed based on expression pattern of all target circRNAs and circRNAs with the top10 up- and down- regulated circRNAs between the two groups (Fig. 2A and 2B). The combined data suggested that the expression of circRNAs in endometrial biopsies with repeated implantation failure is different from that in control tissues. 856 unique circRNAs were significantly altered in women with repeated implantation failure during the implantation window ( $\mathrm{p}<0.05)$. Among them, 423 were significantly up-regulated and 433 were down-regulated. The top 10 up- and down-regulated circRNAs between two groups are listed in Table 1 and Table 2, respectively. Among these, 


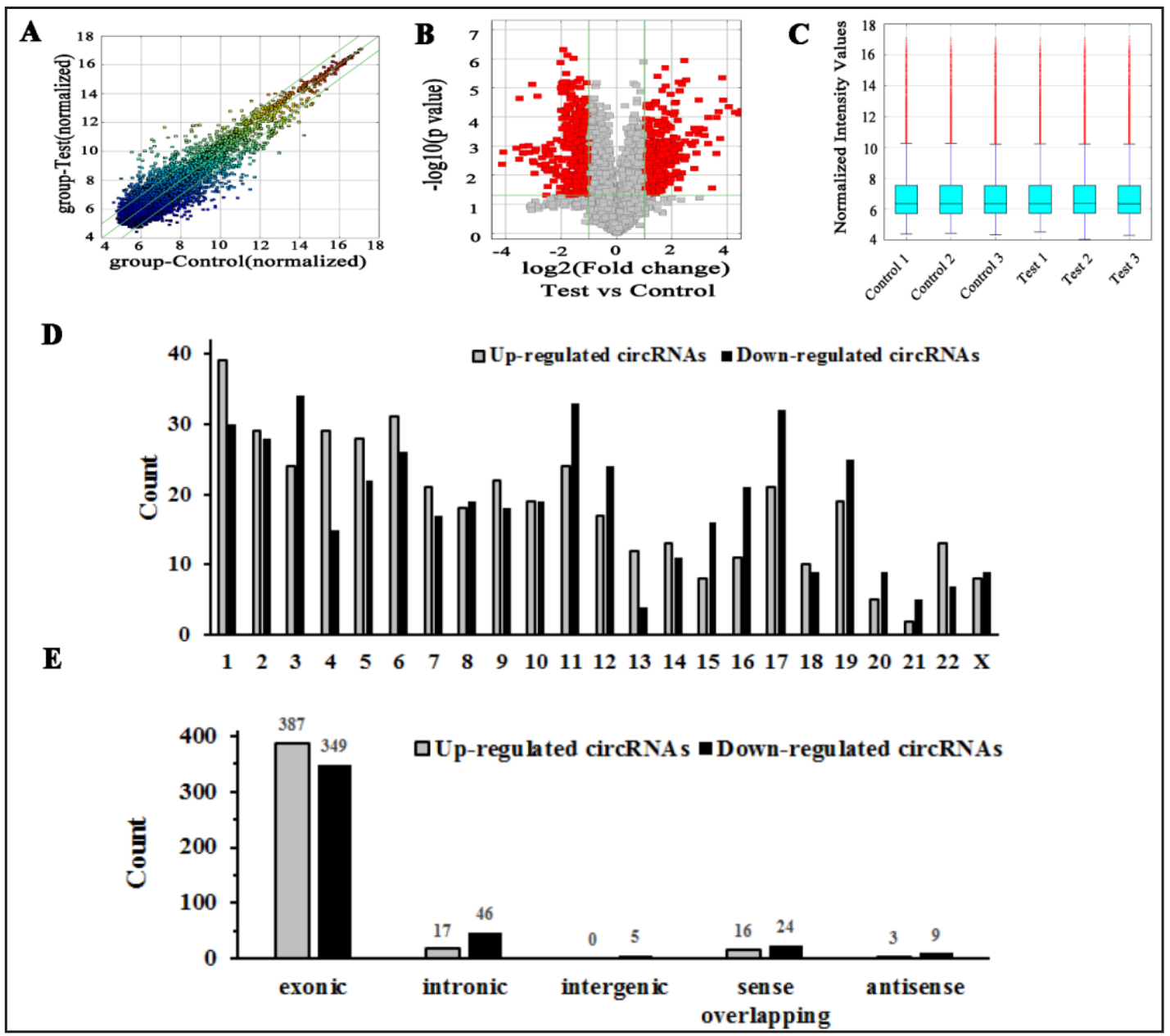

Fig. 1. Bioinformatics analysis of differentially expressed circRNAs in patients with repeated implantation failure. (A) Scatter plot showed the variation of circRNAs in expression in repeated implantation failure (Y-axis) versus the control (X-axis). The values are averaged, normalized signal values (log 2) of circRNA expression in repeated implantation failure. The upper green line to the left indicates a 2.0-fold change of circRNA, while the lower green line indicates a negative fold change of 2.0. After normalization, the distributions of $\log 2$ ratios among samples were nearly the same. (B) Volcano plot showed the differential circRNA expression in repeated implantation failure. Red squares mark differentially expressed circRNAs in repeated implantation failure versus the control $(\mathrm{p}<0.05)$. The vertical lines demark the fold change values. The right vertical line corresponds to 2-fold up and the left vertical line 2-fold down changes, while the horizontal line marks a p-value of 0.05. (C) Box plot showed the distributions of circRNAs in more direct way. (D) The distribution of differentially expressed circRNAs in human chromosomes. (E) The bar diagram shows the circRNA category. Most of differentially expressed circRNAs originate from the exons. Some are from introns, while, a few are other sources.

the expression levels of hsa_circRNA_103717, hsa_circRNA_103828, hsa_circRNA_100834, hsa_circRNA_070616, hsa_circRNA_100833 were up-regulated and hsa_circRNA_405443, hsa_circRNA_023461, hsa_circRNA_104121, hsa_circRNA_403556, hsa_circRNA_000367 were down-regulated as top 5, respectively.

Validation of the differential expression level of circRNAs using qRT-PCR

To confirm the circRNA microarray profiling expression data, 7 typically differential expression circRNAs (hsa_circRNA_070616, hsa_circRNA_103716, hsa_circRNA_104854, hsa_ circRNA_104001, hsa_circRNA_004183, hsa_circRNA_044353, and hsa_circRNA_404686) 
$\mathbf{A}$

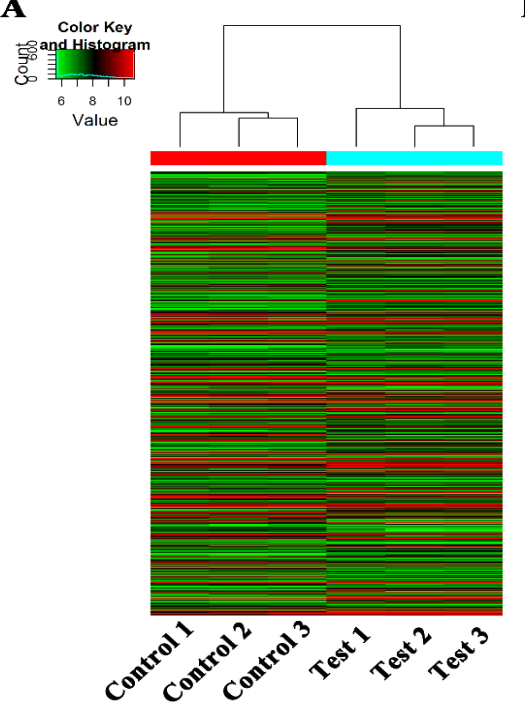

B



Fig. 2. Heat map and hierarchical clustering showing expression values of all target circRNAs and the most up and down regulated circRNAs. Each circRNA was represented by a single row of colored boxes and each sample was represented by a single column. Red strip represents high relative expression and green strip represents low relative expression. (A) Hierarchical cluster analysis of all target circRNAs. (B) Hierarchical cluster analysis of the most 10 up- and down- regulated circRNAs.

Table 1. Comparison of repeated implantation failure (RIF) versus the control for the top 10 up-regulated expression of circRNAs (fold change $>2$ and $\mathrm{p}<0.05$ ) sorted by their fold change $(\mathrm{FC})$

\begin{tabular}{llllllllll}
\hline circRNA & circRNA alias & Symbol & FC & p-value & MRE1 & MRE2 & MRE3 & MRE4 & MRE5 \\
\hline hsa_circRNA_103717 & hsa_cir__0070617 & PAPSS1 & 22.611582 & $6.004 \mathrm{E}-05$ & miR-520a-5p & miR-338-3p & miR-628-5p & miR-525-5p & miR-370-3p \\
hsa_circRNA_103828 & hsa_cir__0072386 & HMGCS1 & 21.492129 & $7.622 \mathrm{E}-05$ & miR-411-5p & miR-625-3p & miR-448 & miR-205-5p & miR-526b-5p \\
hsa_circRNA_100834 & hsa_cir__0022392 & FADS2 & 19.371187 & $6.838 \mathrm{E}-05$ & miR-873-5p & miR-23b-5p & miR-181a-2-3p & miR-93-3p & miR-299-3p \\
hsa_circRNA_070616 & hsa_cir__0070616 & PAPSS1 & 16.533145 & $4.447 \mathrm{E}-05$ & miR-4786-3p & miR-6841-3p & miR-642a-5p & miR-5704 & miR-574-5p \\
hsa_ircRNA_100833 & hsa_cir__0022383 & FADS2 & 14.416911 & $2.634 \mathrm{E}-05$ & miR-765 & miR-495-3p & miR-665 & miR-193b-5p & miR-124-5p \\
hsa_ircRNA_004390 & hsa_cir__004390 & LPAR3 & 14.046012 & $4.443 \mathrm{E}-06$ & miR-2682-5p & miR-6511a-5p & miR-198 & miR-4692 & miR-6780b-5p \\
hsa_circRNA_022382 & hsa_cir_0022382 & FADS2 & 13.382935 & 0.000553 & miR-5586-5p & miR-4726-5p & miR-4640-5p & miR-3138 & miR-8080 \\
hsa_circRNA_103716 & hsa_cir__0006935 & PAPSS1 & 12.879616 & $6.585 \mathrm{E}-05$ & miR-769-5p & miR-574-5p & miR-499a-3p & miR-520a-5p & miR-338-3p \\
hsa_circRNA_005965 & hsa_cir__0005965 & PAPSS1 & 12.29766 & 0.0001026 & miR-6841-3p & miR-3119 & miR-769-5p & miR-5704 & miR-574-5p \\
hsa_circRNA_087856 & hsa_cir__0087856 & RAD23B & 12.224208 & 0.001805 & miR-3692-5p & miR-6511b-5p & miR-4763-3p & miR-4518 & miR-1266-5p \\
\hline
\end{tabular}

Table 2. Comparison of repeated implantation failure (RIF) versus the control for the top 10 down-regulated expression of circRNAs (fold change $>2$ and $\mathrm{p}<0.05$ ) sorted by their fold change ( $\mathrm{FC}$ )

\begin{tabular}{llllllllll}
\hline circRNA & circRNA alias & Symbol & FC & p-value & MRE1 & MRE2 & MRE3 & MRE4 & MRE5 \\
\hline hsa_circRNA_405443 & - & NDE1 & 17.4299507 & 0.004255058 & miR-7109-5p & miR-6780b-5p & miR-548k & miR-6761-5p & miR-4653-5p \\
hsa_circRNA_023461 & hsa_circ_0023461 & ARAP1 & 16.9511166 & 0.001613405 & miR-6720-5p & miR-4512 & miR-6836-5p & miR-766-3p & miR-3918 \\
hsa_circRNA_104121 & hsa_circ_0076767 & TRAM2 & 12.2055249 & 0.001172624 & miR-508-5p & miR-8062 & miR-580-5p & miR-4758-5p & miR-1238-5p \\
hsa_circRNA_403556 & - & LINC00340 & 11.3602467 & 0.002793942 & miR-508-5p & miR-8062 & miR-580-5p & miR-4758-5p & miR-1238-5p \\
hsa_circRNA_000367 & hsa_circ_0000367 & SIAE & 11.3138545 & $2.2919 \mathrm{E}-05$ & miR-331-3p & miR-4646-5p & miR-4797-5p & miR-3919 & miR-3190-3p \\
hsa_circRNA_406237 & - & OXNAD1 & 10.3736491 & 0.001778372 & miR-6868-3p & miR-6739-3p & miR-4639-3p & miR-632 & miR-6886-5p \\
hsa_circRNA_100395 & hsa_circ_0015278 & KLHL20 & 10.2643198 & 0.001008832 & miR-141-3p & miR-588 & miR-660-3p & miR-136-5p & miR-200a-3p \\
hsa_circRNA_102446 & hsa_circ_0049356 & CARM1 & 10.1892307 & 0.001657153 & miR-377-5p & miR-658 & miR-889-5p & miR-23b-5p & let-7i-5p \\
hsa_circRNA_102442 & hsa_circ_0049271 & KEAP1 & 9.0496654 & 0.000975836 & miR-509-5p & miR-593-5p & miR-376b-5p & miR-214-3p & miR-23a-5p \\
hsa_circRNA_001264 & hsa_circ_0000086 & ST6GALNAC3 & 8.8903771 & 0.001667582 & miR-18a-3p & miR-607 & miR-632 & miR-654-3p & miR-10b-3p \\
\hline
\end{tabular}


Liu et al.: CircRNA Expression in Patients with RIF

were selected to be further validated out using another 6 samples with 3 control samples and 3 test samples. Primer sequences were presented in Table 3 and clinical characteristics of the patients is shown as Table 4.

As a result, all of them showed the same trends of up- or downregulation as the microarray data. Particularly, hsa_circRNA_070616, hsa_circRNA_103716, hsa_circRNA_104854 and hsa_circRNA_104001 are significantly up-regulated in patients with repeated implantation failure (Fig. 3A), while hsa_circRNA_004183, hsa_circRNA_044353 and hsa_circRNA_404686 are significantly down-regulated (Fig. 3B). This indicated that the results of qRT-PCR was well consistent with microarray results, demonstrating the high reliability of the microarray expression results.
Table 3. Primer sequences were presented

\begin{tabular}{|c|c|c|}
\hline gene & Primer sequences & PCR product (bp) \\
\hline$\beta$-actin & $\begin{array}{l}\text { F:5'GTGGCCGAGGACTTTGATTG3' } \\
\text { R :5'CCTGTAACAACGCATCTCATATT3' }\end{array}$ & 73 \\
\hline hsa_circRNA_103716 & $\begin{array}{l}\text { F:5'GTGGAACTTCTACAGGAACGGG3' } \\
\text { R:5'TGGGCTTGGTAGGTGACATTG3' }\end{array}$ & 59 \\
\hline hsa_circRNA_070616 & $\begin{array}{l}\text { F:5'TGAACAGAGGGATGTCAAAGG3' } \\
\text { R:5'GGTAGGTGACATTGGTTGCTCT3' }\end{array}$ & 87 \\
\hline hsa_circRNA_104854 & $\begin{array}{l}\text { F:5'ATGTCAATGGGCTATGAACGA3' } \\
\text { R:5'AGTTGTAGCTGGTGCTGGTGT3' }\end{array}$ & 126 \\
\hline hsa_circRNA_104001 & $\begin{array}{l}\text { F:5'CTGGGAGAGAATAAAAAGGAAGA3' } \\
\text { R:5'AAATTTTCTTCTTGTAACTGCTCC3' }\end{array}$ & 119 \\
\hline hsa_circRNA_004183 & $\begin{array}{l}\text { F:5'ATGCCGTCCACGCCAGAC3' } \\
\text { R:5'GCCGACTGCGAGCTATCTGAT3' }\end{array}$ & 87 \\
\hline hsa_circRNA_044353 & $\begin{array}{l}\text { F:5'AAGGAGACCAGCCTAAGAGATGT3' } \\
\text { R:5'CATCCTACACCAAAAGCCCA3' }\end{array}$ & 53 \\
\hline hsa_circRNA_404686 & $\begin{array}{l}\text { F:5'TCTCAGAACAAGAGCGTCCAT3' } \\
\text { R:5'GTAGAGGGGCAACCGGTATT3' }\end{array}$ & 120 \\
\hline
\end{tabular}

Table 4. Clinical characteristics of the patients

\begin{tabular}{cccccc}
$\begin{array}{c}\text { Case } \\
\text { number }\end{array}$ & Age & $\begin{array}{c}\text { Cause of } \\
\text { infertility }\end{array}$ & $\begin{array}{c}\text { Year of } \\
\text { infertility }\end{array}$ & $\begin{array}{c}\text { Total number } \\
\text { of cycles }\end{array}$ & $\begin{array}{c}\text { Endometrial } \\
\text { thickness(cm) }\end{array}$ \\
\hline 1 & 34 & Tubal factor & 3 & 3 & 1.0 \\
2 & 36 & Tubal factor & 14 & 4 & 1.0 \\
3 & 28 & Tubal factor & 7 & 3 & 1.0 \\
4 & 33 & Tubal factor & 7 & 3 & 1.0 \\
5 & 33 & Tubal factor & 5 & 3 & 1.0 \\
6 & 32 & Tubal factor & 3 & 3 & 1.0 \\
\hline
\end{tabular}

MRE analysis of differentially expressed circRNAs in patients with repeated implantation failure

Because the circRNA may affect the development of repeated implantation failure through the miRNAs, interactions between circRNAs and their target miRNAs were predicted with arraystar's homemade miRNA target prediction software. Table 1 and Table 2 displayed the potential complementary binding miRNAs of top 10 up-regulated and down-regulated circRNA, respectively. Furthermore, endometrial receptive relevant miRNAs previously described as differentially expressed in pa-

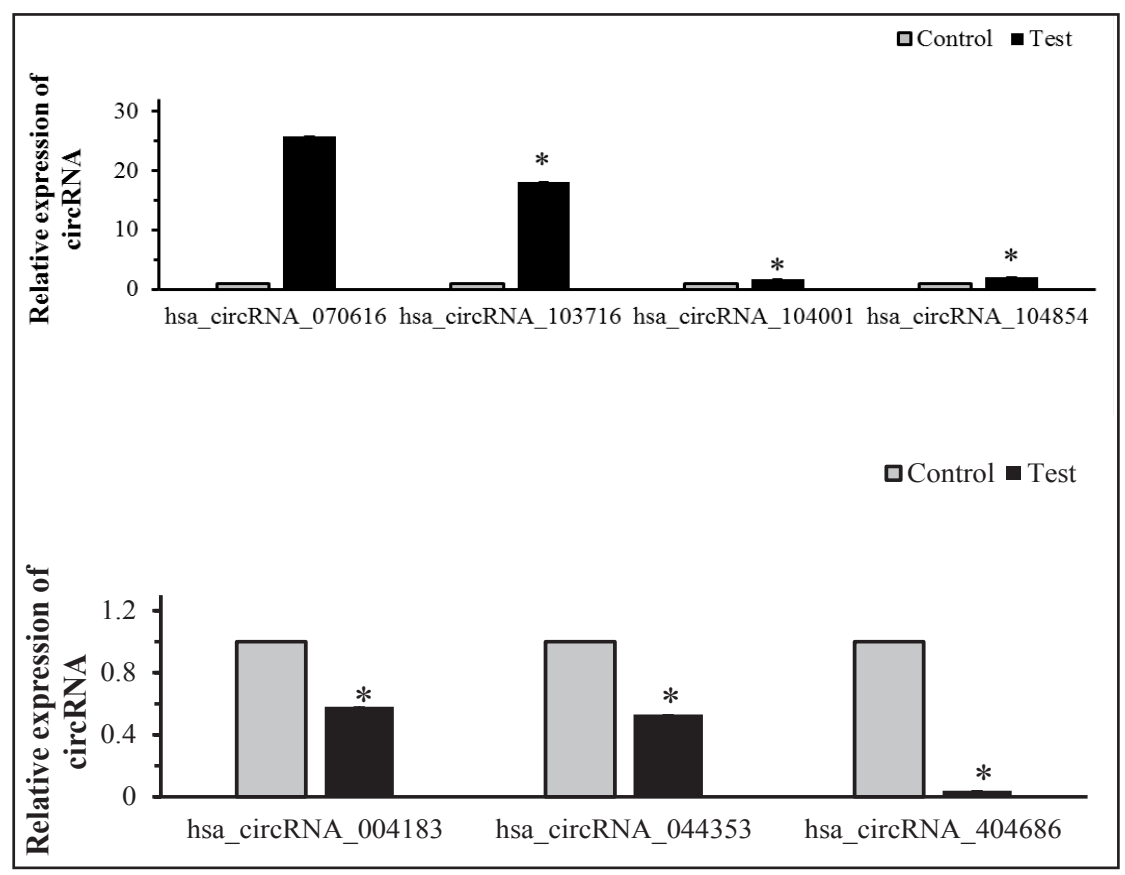

Fig. 3. Quantitative RT-PCR validation for five circRNAs from the microarray data. Fold changes were calculated by the $2^{\wedge}(-\Delta \Delta \mathrm{Ct})$ method and $\beta$-actin expression is used as a control. (A) The expression level of up-regulated circRNAs. (B) The expression level of down-regulated circRNAs. 
Liu et al.: CircRNA Expression in Patients with RIF

Table 5. Endometrial receptive relevant miRNAs previously described as differentially expressed in patients with repeated implantation failure and corresponding circRNA matches

\begin{tabular}{|c|c|c|}
\hline microRNA & circRNA match & Author \\
\hline \multicolumn{3}{|c|}{ Up-regulated microRNA } \\
\hline hsa-miR-145-5p & $\begin{array}{l}\text { hsa_circRNA_102324, hsa_circRNA_101206, hsa_circRNA_091419, hsa_circRNA_101139, } \\
\text { hsa_circRNA_003484, hsa_circRNA_102293, hsa_circRNA_100422, hsa_circRNA_400759, } \\
\text { hsa_circRNA_031896, hsa_circRNA_102910, hsa_circRNA_104821 }\end{array}$ & Ariel et al.[17] \\
\hline hsa-miR-27b-3p & hsa_circRNA_104503, hsa_circRNA_101876, hsa_circRNA_003653 & Ariel et al. [17] \\
\hline hsa-miR-652 & hsa_circRNA_104293, hsa_circ_0086419, hsa_circRNA_048474 & Ariel et al. [17] \\
\hline hsa-miR-195 & hsa_circRNA_101853, hsa_circRNA_101852, hsa_circRNA_103457, hsa_circRNA_102747 & Ariel et al. [17] \\
\hline hsa-miR-342-3p & hsa_circRNA_001459, hsa_circRNA_102485, hsa_circRNA_000966 & Ariel et al. [17] \\
\hline hsa-miR-150-5p & hsa_circRNA_001405 & Ariel et al. [17] \\
\hline hsa-miR-23b-3p & hsa_circRNA_007059 & Ariel et al. [17] \\
\hline hsa-miR-139-5p & hsa_circRNA_104168 & Ariel et al. [17] \\
\hline hsa-miR-99a-5p & hsa_circRNA_102646 & Ariel et al. [17] \\
\hline hsa-miR-1972 & hsa_circRNA_405698, hsa_circRNA_006290 & Youngsok et al. [18] \\
\hline hsa-miR-29b-1-5p & $\begin{array}{l}\text { hsa_circRNA_101139, hsa_circRNA_101787, hsa_circRNA_104748, hsa_circRNA_104503, } \\
\text { hsa_circRNA_101184, hsa_circRNA_104392, hsa_circRNA_101356, hsa_circRNA_102378, } \\
\text { hsa_circRNA_102039, hsa_circRNA_100776, hsa_circRNA_406051, hsa_circRNA_102923, } \\
\text { hsa_circRNA_406866, hsa_circRNA_104101, hsa_circRNA_104664, hsa_circRNA_008761, } \\
\text { hsa_circRNA_400890 }\end{array}$ & Youngsok et al. [18] \\
\hline hsa-miR-1246 & hsa_circRNA_400850, hsa_circRNA_105055 & Youngsok et al. [18] \\
\hline hsa-miR-1273f & hsa_circRNA_406326 & Youngsok et al. [18] \\
\hline hsa-miR-363-3p & hsa_circRNA_001729 & Youngsok et al. [18] \\
\hline hsa-miR-654-3p & hsa_circRNA_001264, hsa_circRNA_101000, hsa_circRNA_103754, hsa_circRNA_103139 & Youngsok et al. [18] \\
\hline hsa-miR-34b-3p & hsa_circRNA_405698 & Youngsok et al. [18] \\
\hline hsa-miR-138-1-3p & hsa_circRNA_027612 & Youngsok et al. [18] \\
\hline hsa-miR-654-3p & hsa_circRNA_101000, hsa_circRNA_001264 & \\
\hline hsa-miR-346 & hsa_circRNA_102324, hsa_circRNA_104293, hsa_circRNA_100834 & Youngsok et al. [18] \\
\hline \multicolumn{3}{|c|}{ Down-regulated microRNA } \\
\hline hsa-miR-32-5p & hsa_circRNA_400322 & Ariel et al. [17] \\
\hline hsa-miR-628-5p & $\begin{array}{l}\text { hsa_circRNA_103717, hsa_circRNA_104000, hsa_circRNA_103670, hsa_circRNA_103715, } \\
\text { hsa_circRNA_104267 }\end{array}$ & Ariel et al. [17] \\
\hline hsa-miR-874-5p & hsa_circRNA_101805, hsa_circRNA_104143, hsa_circRNA_103221, hsa_circRNA_102451 & Ariel et al. [17] \\
\hline hsa-miR-3187-3p & hsa_circRNA_403782 & Youngsok et al. [18] \\
\hline hsa-miR-21-3p & $\begin{array}{l}\text { hsa_circRNA_100186, hsa_circRNA_102452, hsa_circRNA_076989, hsa_circRNA_102747, } \\
\text { hsa_circRNA_102434, hsa_circRNA_100604 }\end{array}$ & Youngsok et al. [18] \\
\hline hsa-miR-181c-5p & hsa_circRNA_102949, hsa_circRNA_102950, hsa_circRNA_101762 & Youngsok et al. [18] \\
\hline hsa-miR-299-3p & hsa_circRNA_008952, hsa_circRNA_100834, hsa_circRNA_102324 & Youngsok et al. [18] \\
\hline hsa-miR-495-3p & hsa_circRNA_100833, hsa_circRNA_104175, hsa_circRNA_100735, hsa_circRNA_104174 & Youngsok et al. [18] \\
\hline hsa-miR-205-5p & hsa_circRNA_103827, hsa_circRNA_103754, hsa_circRNA_103828 & Youngsok et al. [18] \\
\hline hsa-miR-188-5p & hsa_circRNA_100246, hsa_circRNA_400994 & Youngsok et al. [18] \\
\hline hsa-miR-551b-5p & hsa_circRNA_101609, hsa_circRNA_103706, hsa_circRNA_104536 & Youngsok et al. [18] \\
\hline hsa-miR-485-5p & $\begin{array}{l}\text { hsa_circRNA_048728, hsa_circRNA_009181, hsa_circRNA_102001, hsa_circRNA_020596, } \\
\text { hsa_circRNA_102056, hsa_circRNA_103339, hsa_circRNA_103219, hsa_circRNA_104913, } \\
\text { hsa_circRNA_001225, hsa_circRNA_100790, hsa_circRNA_104165, hsa_circRNA_102597, } \\
\text { hsa_circRNA_102598, hsa_circRNA_101979, hsa_circRNA_074491, hsa_circRNA_100935, } \\
\text { hsa_circRNA_100934 }\end{array}$ & Youngsok et al. [18] \\
\hline
\end{tabular}

tients with repeated implantation failure $[17,18]$ and corresponding circRNA match which are differently expressed in our circRNA microarray results are shown in Table 5. The 2D structure were compiled to show the MRE sequence of validated circRNA and the target miRNA seed type (Fig. 4). 


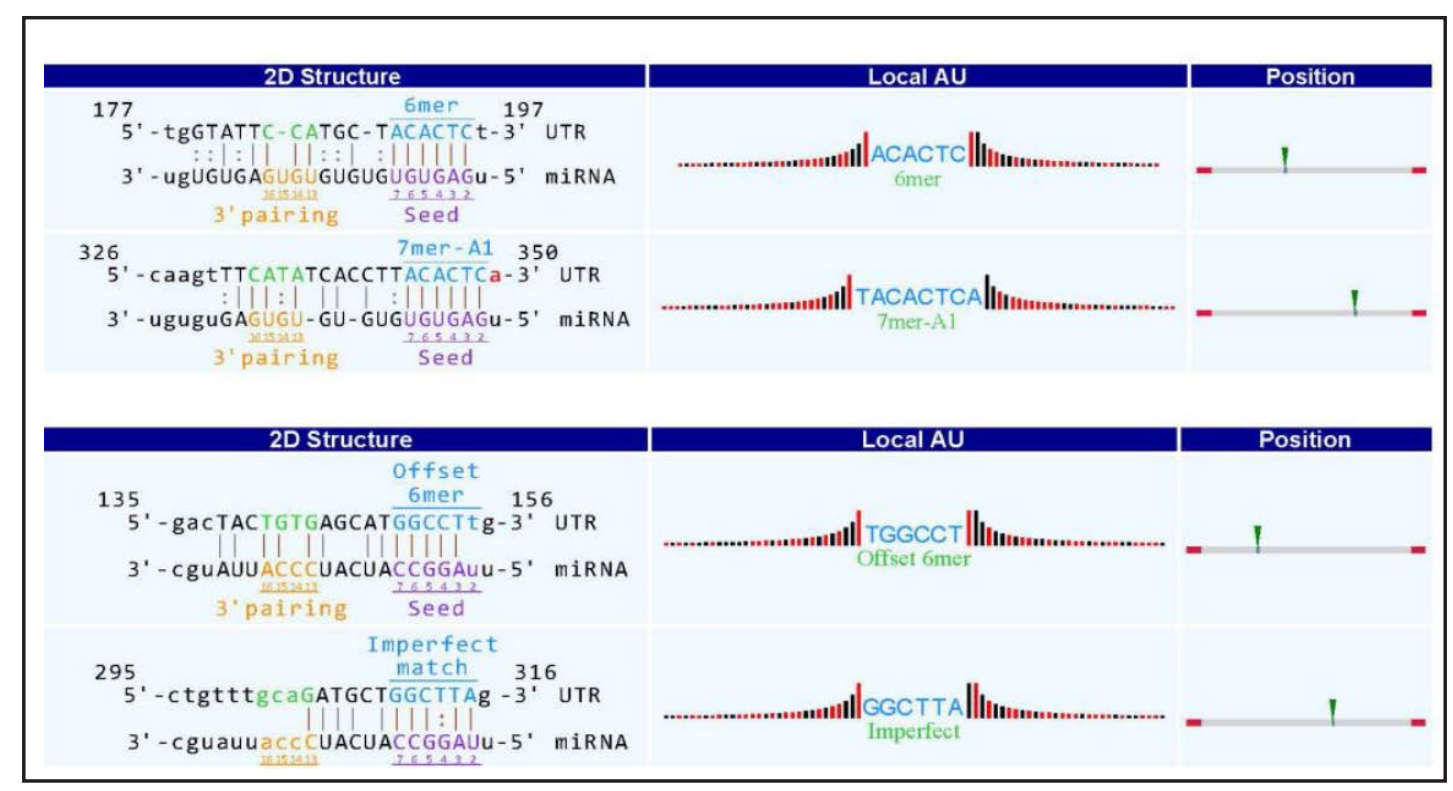

Fig. 4. Binding sites of miR-574-5p in 3'UTR of circRNA_103716 and circRNA_070616.

\section{Discussion}

In the present study, the differentially expressed profiles of circRNAs in repeated implantation failure patients were analyzed and validated. To our knowledge, this is the first study to evaluate the differential expression of circRNAs in the endometrium from patients with repeated implantation failures, indicating that the repeated implantation failureassociated circRNAs could be exploited as new candidates for diagnosis and treatment of embryo implantation failures.

CircRNAs are recently discovered as unique class of endogenous non-coding RNAs that can affect the expression of the target genes of miRNA by binding and preventing miRNAs expression with multiple miRNA binding sites [8-10]. It has been reported that circRNAs participate in many essential biological processes and play important role as miRNA sponge [19-23]. However, there is no studies to date have reported the effects of circRNAs in patients with repeated implantation failure.

In this study, there were 423 circRNAs significantly up-regulated and 433 circRNAs significantly down-regulated in endometrial tissue from patients with repeated implantation failure compared to normal controls. In which, hsa_circRNA_103717, hsa_circRNA_103828, hsa_circRNA_100834, hsa_circRNA_070616, hsa_circRNA_100833 were up-regulated and hsa_circRNA_405443,

hsa_circRNA_023461,hsa_circRNA_104121, hsa_circRNA_403556, hsa_circRNA_000367 were down-regulated as top 5, respectively. Furthermore, the differential expression of circRNA whose matched miRNA is related with endometrial receptive was analyzed. The up-regulated hsa_circRNA_070616, hsa_circRNA_103716, hsa_circRNA_104854, hsa_circRNA_104001 and down-regulated hsa_circRNA_004183, hsa_circRNA_044353 and hsa_circRNA_404686 were validated by qRT-PCR analysis. Consistent results were observed, indicating that the altered expression levels of circRNAs may be related to the involvement of repeated implantation failure. Most of these differential circRNAs (hsa_circRNA_070616, hsa_circRNA_103716, hsa_circRNA_104854, hsa_circRNA_004183, hsa_circRNA_044353) are reported expressed in the mammalian brain [24]. Li et al. [25] have reported that hsa_circRNA_004183 play an important regulatory role in hepatic steatosis, but there is no report about the detailed mechanism. Hsa_circRNA_070616 and hsa_circRNA_103716 are selected for further analysis 
because they are both predicted as sponge of hsa-miR-574-5p. The previous study about the function of hsa-miR-574-5p are mainly focus on the invasion and metastasis of small/ non-small cell lung cancer [26, 27]. On the contrary, Cui et al. [28] have reported that hsamiR-574-5p suppress the liver metastasis of colorectal cancer by reducing the expression of MACC-1. The study of Ma et al. [29] have reported that overexpression of miR-574-5p inhibit the growth and metastasis in cervical cancer. Notably, Zhang et al. [30] have reported that hsa-miR-574-5p play an important role in hyperplastic endometria and could be a biomarker as estrogen over-exposure. A few studies have reported that supplement of estrogen may improve the thickness of endometrial of patients with repeated implantation failure [31,32]. However, the relationship between hsa-miR-574-5p and endometrial receptive, the network of hsa_circRNA_070616/hsa_circRNA_103716- hsa-miR-574-5p-mRNA need to be further studied. CircRNAs were believed to function as miRNA sponge. Our results implied that it is worthwhile to further investigate these novel dysregulated circRNAs as miRNA sponges and their potential biological functions in the development of endometrial receptive with patients of repeated implantation failure.

\section{Conclusion}

In conclusion, for the first time, our study revealed a unique set of circRNA expression signatures in endometrial tissue from patients with repeated implantation failure. Furthermore, the potential roles of dysregulated circRNAs (hsa_circRNA_070616/hsa circRNA_103716) were investigated by bioinformatic analysis and they may function as sponge of hsa-miR-574-5p. In summary, the present study could improve our knowledge of the molecular mechanism of repeated implantation failure and would be helpful for further studies on diagnostic, therapeutic and functional of circRNAs in patients with repeated implantation failure. However, differential expression of circRNA related with repeated implantation failure should be validated further in larger studies and the detailed interaction mechanisms between hsa_circRNA_070616/hsa_circRNA_103716 and its target miRNAs are preliminary and need to be validated in future.

\section{Acknowledgements}

We thank all the donors who participated in work. This study was supported by the National Natural Science Foundation of China (81401261), the Fundamental Research Funds for the Central Universities (lzujbky-2015- 37) and MERCK CREAT Project (MerckSerono CREATE-2016154).

Lin Liu and Xuehong Zhang conceived and designed the experiments; Lin Liu, Yiqing Wang and Feng Yue performed the experiments; Liyan Wang and Panpan Jin analyzed the data; Lifei Li and Xiaoling Ma contributed reagents/materials/analysis tools; Lin Liu wrote the paper.

\section{Disclosure Statement}

The authors declare no conflict of interest.

\section{References}

\footnotetext{
1 Liu L, Lian J, Zhang H, Tian H, Liang M, Yin M, Sun F: MicroRNA-302a sensitizes testicular embryonal carcinoma cells to cisplatin-induced cell death. J Cell Physiol 2013;228:2294-2304.
} 


\section{Cellular Physiology \begin{tabular}{l|l|l} 
and Biochemistry Published 10.1159/000484887 & $\begin{array}{l}\text { (c) } 2017 \text { The Author(s). Published by S. Karger AG, Basel } \\
\text { www.karger.com/cpb }\end{array}$
\end{tabular}

Liu et al.: CircRNA Expression in Patients with RIF

-2 Chung TW, Park MJ, Kim HS, Choi HJ, Ha KT: Integrin alphaVbeta3 and alphaVbeta5 are required for leukemia inhibitory factor-mediated the adhesion of trophoblast cells to the endometrial cells. Biochem Biophys Res Commun 2016;469:936-940.

3 Bastu E, Mutlu MF, Yasa C, Dural O, Nehir Aytan A, Celik C, Buyru F, Yeh J: Role of Mucin 1 and Glycodelin A in recurrent implantation failure. Fertil Steril 2015;103:1059-1064 e1052.

4 Ou C, Li G: Long non-coding RNA TUG1: a novel therapeutic target in small cell lung cancer. J Thorac Dis 2017;9:E644-E645.

-5 Li W, Li H, Zhang L, Hu M, Li F, Deng J, An M, Wu S, Ma R, Lu J, Zhou Y: Long non-coding RNA LINC00672 contributes to $\mathrm{p} 53$ protein-mediated gene suppression and promotes endometrial cancer chemosensitivity. J Biol Chem 2017;292:5801-5813.

-6 Montagnana M, Benati M, Danese E, Giudici S, Perfranceschi M, Ruzzenenete O, Salvagno GL, Bassi A, Gelati M, Paviati E, Guidi GC, Franchi M, Lippi G: Aberrant MicroRNA Expression in Patients With Endometrial Cancer. Int J Gynecol Cancer 2017;27:459-466.

7 Nothnick WB: MicroRNAs and Endometriosis: Distinguishing Drivers from Passengers in Disease Pathogenesis. Semin Reprod Med 2017;35:173-180.

-8 Li H, Hao X, Wang H, Liu Z, He Y, Pu M, Zhang H, Yu H, Duan J, Qu S: Circular RNA Expression Profile of Pancreatic Ductal Adenocarcinoma Revealed by Microarray. Cell Physiol Biochem 2016;40:1334-1344. Wu HJ, Zhang CY, Zhang S, Chang M, Wang HY: Microarray Expression Profile of Circular RNAs in Heart Tissue of Mice with Myocardial Infarction-Induced Heart Failure. Cell Physiol Biochem 2016;39:205-216.

10 Qian Y, Lu Y, Rui C, Qian Y, Cai M, Jia R: Potential Significance of Circular RNA in Human Placental Tissue for Patients with Preeclampsia. Cell Physiol Biochem 2016;39:1380-1390.

-11 Hansen TB, Jensen TI, Clausen BH, Bramsen JB, Finsen B, Damgaard CK, Kjems J: Natural RNA circles function as efficient microRNA sponges. Nature 2013;495:384-388.

-12 Lukiw WJ: Circular RNA (circRNA) in Alzheimer's disease (AD). Front Genet 2013;4:307.

13 Li P, Chen S, Chen H, Mo X, Li T, Shao Y, Xiao B, Guo J: Using circular RNA as a novel type of biomarker in the screening of gastric cancer. Clin Chim Acta 2015;444:132-136.

14 Lin SP, Ye S, Long Y, Fan Y, Mao HF, Chen MT, Ma QJ: Circular RNA expression alterations are involved in OGD/R-induced neuron injury. Biochem Biophys Res Commun 2016;471:52-56.

15 Ghosal S, Das S, Sen R, Basak P, Chakrabarti J: Circ2Traits: a comprehensive database for circular RNA potentially associated with disease and traits. Front Genet 2013;4:283.

16 Dang Y, Yan L, Hu B, Fan X, Ren Y, Li R, Lian Y, Yan J, Li Q Zhang Y, Li M, Ren X, Huang J, Wu Y, Liu P, Wen L, Zhang C, Huang Y, Tang F, Qiao J: Tracing the expression of circular RNAs in human pre-implantation embryos. Genome Biol 2016;17:130.

17 Revel A, Achache H, Stevens J, Smith Y, Reich R: MicroRNAs are associated with human embryo implantation defects. Hum Reprod 2011;26:2830-2840.

18 Choi Y, Kim HR, Lim EJ, Park M, Yoon JA, Kim YS, Kim EK, Shin JE, Kim JH, Kwon H, Song H, Choi DH: Integrative Analyses of Uterine Transcriptome and MicroRNAome Reveal Compromised LIF-STAT3 Signaling and Progesterone Response in the Endometrium of Patients with Recurrent/Repeated Implantation Failure (RIF). PLoS One 2016;11:e0157696.

19 Li J, Yang J, Zhou P, Le Y, Zhou C, Wang S, Xu D, Lin HK, Gong Z: Circular RNAs in cancer: novel insights into origins, properties, functions and implications. Am J Cancer Res 2015;5:472-480.

-20 Bachmayr-Heyda A, Reiner AT, Auer K, Sukhbaatar N, Aust S, Bachleitner-Hofmann T, Mesteri I, Grunt TW, Zeillinger R, Pils D: Correlation of circular RNA abundance with proliferation--exemplified with colorectal and ovarian cancer, idiopathic lung fibrosis, and normal human tissues. Sci Rep 2015;5:8057.

21 Hansen TB, Kjems J, Damgaard CK: Circular RNA and miR-7 in cancer. Cancer Res 2013;73:5609-5612.

-22 Peng L, Yuan XQ Li GC: The emerging landscape of circular RNA ciRS-7 in cancer (Review). Oncol Rep 2015;33:2669-2674.

23 Caiment F, Gaj S, Claessen S, Kleinjans J: High-throughput data integration of RNA-miRNA-circRNA reveals novel insights into mechanisms of benzo [a]pyrene-induced carcinogenicity. Nucleic Acids Res 2015;43:2525-2534.

24 Rybak-Wolf A, Stottmeister C, Glazar P, Jens M, Pino N, Giusti S, Hanan M, Behm M, Bartok O, Ashwal-Fluss R, Herzog M, Schreyer L, Papavasileiou P, Ivanov A, Ohman M, Refojo D, Kadener S, Rajewsky N: Circular RNAs in the Mammalian Brain Are Highly Abundant, Conserved, and Dynamically Expressed. Mol Cell 2015;58:870-885. 


\section{Cellular Physiology Cell Physiol Biochem 2017;44:303-313 \begin{tabular}{l|l|l|l} 
DOI: 10.1159/000484887 & $\begin{array}{l}\text { O 2017 The Author(s). Published by S. Karger AG, Basel } \\
\text { www.karger.com/cpb }\end{array}$ \\
\cline { 2 - 3 }
\end{tabular}

-25 Guo XY, He CX, Wang YQ, Sun C, Li GM, Su Q, Pan Q, Fan JG: Circular RNA Profiling and Bioinformatic Modeling Identify Its Regulatory Role in Hepatic Steatosis. Biomed Res Int 2017;2017:5936171.

-26 Zhou R, Zhou X, Yin Z, Guo J, Hu T, Jiang S, Liu L, Dong X, Zhang S, Wu G: MicroRNA-574-5p promotes metastasis of non-small cell lung cancer by targeting PTPRU. Sci Rep 2016;6:35714.

-27 Zhou R, Zhou X, Yin Z, Guo J, Hu T, Jiang S, Liu L, Dong X, Zhang S, Wu G: Tumor invasion and metastasis regulated by microRNA-184 and microRNA-574-5p in small-cell lung cancer. Oncotarget 2015;6:4460944622.

28 Cui Z, Tang J, Chen J, Wang Z: Hsa-miR-574-5p negatively regulates MACC-1 expression to suppress colorectal cancer liver metastasis. Cancer Cell Int 2014;14:47.

29 Ma DL, Li JY, Liu YE, Liu CM, Li J, Lin GZ, Yan J: Influence of continuous intervention on growth and metastasis of human cervical cancer cells and expression of RNAmiR-574-5p. J Biol Regul Homeost Agents 2016;30:91-102.

30 Zhang R, He Y, Zhang X, Xing B, Sheng Y, Lu H, Wei Z: Estrogen receptor-regulated microRNAs contribute to the BCL2/BAX imbalance in endometrial adenocarcinoma and precancerous lesions. Cancer Lett 2012;314:155-165.

-31 Kumar P, Mahajan S: Preimplantation and postimplantation therapy for the treatment of reproductive failure. J Hum Reprod Sci 2013;6:88-92.

-32 Shen MS, Wang CW, Chen CH, Tzeng CR: New horizon on successful management for a woman with repeated implantation failure due to unresponsive thin endometrium: use of extended estrogen supplementation. J Obstet Gynaecol Res 2013;39:1092-1094. 